\title{
Nuclear Saturation with in-Medium Meson Exchange Interactions
}

\author{
R. Rapp ${ }^{1}$, R. Machleidt ${ }^{2}$, J.W. Durso ${ }^{1,3}$, and G.E. Brown ${ }^{1}$ \\ ${ }^{1}$ Department of Physics, SUNY at Stony Brook, Stony Brook NY 11794-3800, U.S.A. \\ ${ }^{2}$ Department of Physics, University of Idaho, Moscow, ID 83844, U.S.A. \\ ${ }^{3}$ Physics Department, Mount Holyoke College, South Hadley, MA 01075, U.S.A.
}

(May 1997)

\begin{abstract}
We show that the assumption of dropping meson masses together with conventional many-body effects, implemented in the relativistic Dirac-Brueckner formalism, explains nuclear saturation. We use a microscopic model for correlated $2 \pi$ exchange and include the standard many-body effects on the in-medium pion propagation, which initially increase the attractive nucleon-nucleon $(N N)$ potential with density. For the vector meson exchanges in both the $\pi \pi$ and $N N$ sector, we assume Brown-Rho scaling which - in concert with 'chiral' $\pi \pi$ contact interactions - reduces the attraction at higher densities.
\end{abstract}

PACS numbers: 13.75.Lb, 13.75.Cs, 21.30.Fe, 21.65.+f 
The description of the ground state properties of nuclear matter in terms of an underlying microscopic nucleon-nucleon $(N N)$ interaction has been one of the major challenges in modern nuclear physics (for a review see, e.g., [1]). Nonrelativistic many-body approaches, such as Brueckner-Hartree-Fock (BHF), resulted in the famous Coester band [2], which does not meet the empirical saturation point. However, including the effective in-medium nucleon mass in the relativistic Dirac structure of the nucleon, as done in the so-called DiracBHF (DBHF) approach [1],3], generates additional saturation, resolving the aforementioned discrepancy. In these frameworks, the mesonic degrees of freedom are usually 'frozen'; i.e., no medium effects are applied to mesons. This is a natural, but unjustified, simplification.

In the next simplest scenario, in analogy to the inmedium nucleon mass (or by arguments based on scale invariance [4]), one would assume that also the masses of the exchanged mesons (except for the pion, which is protected by its Goldstone nature) depend on the nuclear density. However, if the meson masses decrease at the same rate, saturation cannot be reached at the empirical density, since the enhanced attraction in the effective $\sigma(550)$ exchange overwhelms the corresponding increase in repulsion due to $\omega(782)$ exchange [5] 7].

More microscopically, the $\sigma(550)$ is understood as a correlated pair of pions in a relative s-wave (the strong attraction in the s-wave $\pi \pi$ interaction being chiefly due to $t$-channel $\rho$ exchange). Therefore, as a consequence of the well established p-wave pion polarization in nuclear matter due to $\Delta$-hole and nucleon-hole excitations, the spectral distribution of the 'effective' $\sigma$ meson undergoes an appreciable reshaping: the softening of the in-medium pion dispersion relation leads to a considerable shift of strength to lower energies [8,9]. This, in turn, affects the $N N$ interaction in the nuclear environment, causing a marked increase in attraction. Qualitatively, this effect is comparable to that of a reduced ' $\sigma(550)$ ' mass in the one boson exchange picture of the $N N$ potential. However, additionally accounting for a density dependence in a chirally symmetric $\pi \pi$ interaction will slow down the increase of attraction, particularly at densities above $\rho_{0}$, thus improving saturation.

In this note we will show that a unified treatment of 'conventional' many-body effects coupled with the assumption of decreasing meson masses, implemented in a DBHF framework, does indeed lead to reasonable saturation properties of nuclear matter.

First let us briefly review our model for the correlated two-pion exchange in the nucleon-nucleon potential 10,11. We employ a chirally improved version 9] of an earlier meson exchange model [12] for the free $\pi \pi$ interaction. Its dominant contribution at low energies stems from $t$-channel exchange of $\rho$ mesons which, in the scalar-isoscalar ( $\left.~ \sigma{ }^{\prime}\right)$ channel, provides sufficient attraction to form the broad resonance-like structure around $E_{\pi \pi} \simeq 500 \mathrm{MeV}$. However the inclusion of $\pi \pi$ contact interactions, dictated by chiral symmetry 13] and repulsive in nature, turned out to be crucial in avoiding unrealistic $\pi \pi$ bound states in the nuclear environment 8.9] while still enabling a satisfactory description of the free $\pi \pi$ scattering data [9:14. The $\pi \pi$ interaction is then used to calculate a scattering amplitude for the $N \bar{N} \rightarrow \pi \pi \rightarrow N \bar{N}$ reaction [10,11]. Schematically,

$$
\begin{aligned}
M_{N \bar{N}}\left(t^{\prime}\right) & =\tau_{B}^{*} G_{\pi \pi}^{0} \tau_{B}+\tau_{B}^{*} G_{\pi \pi}^{0} M_{\pi \pi} G_{\pi \pi}^{0} \tau_{B} \\
& \equiv M_{N \bar{N}}^{\text {bare }}\left(t^{\prime}\right)+M_{N \bar{N}}^{r e s c a t}\left(t^{\prime}\right),
\end{aligned}
$$

where $\tau_{B}$ denotes the transition Born amplitudes for $N \bar{N} \rightarrow \pi \pi$ (consisting of nucleon and $\Delta$ exchange) and $M_{\pi \pi}, G_{\pi \pi}$ the $\pi \pi$ scattering amplitude and $2 \pi$ propagator, respectively. In the so-called pseudophysical region (i.e., for energies $\sqrt{t^{\prime}}$ well below the nucleon-antinucleon threshold), it is sufficient to consider $\pi \pi / K \bar{K}$ intermediate states (contained in our model for $M_{\pi \pi}$ ). Again, the full transition amplitudes,

$$
\tau=\tau_{B}+\tau_{B} G_{\pi \pi} M_{\pi \pi},
$$

are in good agreement with quasiempirical information obtained by Höhler et al. [15]. Finally, the $I=J=0 N \bar{N}$ amplitude is related via a dispersion relation to the correlated $2 \pi$ exchange potential in the $N N$ channel as

$$
\begin{gathered}
V_{N N}^{2 \pi, \text { corr }}(t)=-\frac{1}{\pi} \int_{4 m_{\pi}^{2}}^{t_{c}} d t^{\prime} \frac{\eta_{00}\left(t^{\prime}\right)}{t^{\prime}-t}, \\
\eta_{00}\left(t^{\prime}\right)=\frac{4 m_{N}^{2}}{t^{\prime}-4 m_{N}^{2}} \frac{3}{4 \pi} \operatorname{Im}_{N \bar{N}}^{00, \text { rescat }}\left(t^{\prime}\right),
\end{gathered}
$$

where $t=\left(k^{\prime}-k\right)^{2} \equiv k^{\prime \prime 2}$ is the 4-momentum transfer between the two nucleons. For practical purposes, we extract a coordinate space potential by taking the quasistatic limit, $k^{\prime \prime} \simeq-k^{\prime \prime}$, and Fourier transforming (3):

$$
V_{N N}^{2 \pi, c o r r}(r)=-\frac{1}{\pi} \int_{4 m_{\pi}^{2}}^{t_{c}} d t^{\prime} \eta_{00}\left(t^{\prime}\right) \frac{\exp \left(-\sqrt{t^{\prime}} r\right)}{r} .
$$

Let us now turn to the medium modifications. In order to preserve the analogy with the one boson exchange picture of the $N N$ interaction, we regard the correlated $2 \pi$ contribution as an 'effective $\sigma$ '. That means that we restrict the effects of the nuclear environment in Eq. (1) to the $\pi \pi$ amplitude $M_{\pi \pi}$. The latter is obtained by solving a Lippmann-Schwinger-type equation

$$
M_{\pi \pi}=V_{\pi \pi}+V_{\pi \pi} G_{\pi \pi} M_{\pi \pi},
$$

where medium effects are induced in both $G_{\pi \pi}$ (through a change in the pion dispersion relation) and in the $\pi \pi$ 
interaction kernel $V_{\pi \pi}$. As has been shown in Ref. [16], the restriction to medium effects in $G_{\pi \pi}$ yields $N N$ potentials which will not be compatible with nuclear saturation. However, as was conjectured in Ref. [16], the inclusion of density-dependent meson masses and coupling constants in the $\pi \pi$ interaction kernel might provide a remedy to this problem. Here we work at mean field level, changing only $f_{\pi}^{*}$ and masses according to

$$
\Phi(\rho)=\left(1-C \rho / \rho_{0}\right)=\frac{f_{\pi}^{*}}{f_{\pi}}=\frac{m^{*}}{m}=\frac{\Lambda^{*}}{\Lambda} \quad \text { etc. }
$$

with the scaling factor taken to be $C=0.15$ (which is in line with QCD sum rule analyses [17], and has become known as Brown-Rho (BR) scaling (4). Explicitly, the following quantities in the $\pi \pi$ interaction kernel are subject to this medium dependence (indicated by an asterisk):

(i) the mass $m_{\rho}^{*}$ and formfactor cutoff $\Lambda_{\rho}^{*}$ of the $t$ channel $\rho$ exchange;

(ii) the pion decay constant $f_{\pi}^{*}$ and $m_{\rho}^{*}$, both entering the $\pi \pi$ contact interactions.

Since the KSFR relation is supposed to remain valid in the medium,

$$
2 g_{\pi \pi \rho}^{2}\left(f_{\pi}^{*}\right)^{2}=\left(m_{\rho}^{*}\right)^{2}
$$

the $\rho \pi \pi$ coupling constant is not affected. The same holds for masses and cutoffs related to the pion due to its Goldstone boson nature. For consistency, corresponding modifications are also applied in the single-pion self energy, $\Sigma_{\pi}$, which is responsible for the many-body effects in the (uncorrelated) $2 \pi$ propagator $G_{\pi \pi}$. It is calculated in terms of standard p-wave particle-hole $\left(N N^{-1}\right.$ and $\Delta N^{-1}$ ) excitations [18]:

$$
\Sigma_{\pi}(\omega, k)=-z_{\pi}^{2} k^{2} \chi(\omega, k) .
$$

The pion susceptibility, $\chi$, contains short-range correlation effects between particle and hole parametrized by Migdal parameters $g^{\prime}$. Since the dynamical origin of the latter is partly due to vector meson exchange we employ a density dependent form

$$
\begin{aligned}
& \left(g_{N N}^{\prime}\right)^{*}=0.65+C \rho / \rho_{0} \\
& \left(g_{N \Delta}^{\prime}\right)^{*}=\left(g_{\Delta \Delta}^{\prime}\right)^{*}=0.35+C \rho / \rho_{0},
\end{aligned}
$$

which is somewhat weaker than that suggested in Ref. [19], where it was erroneously assumed that the anomalous $\rho N N$ coupling through $\kappa_{V}$ should be enhanced by the factor $\left(m_{N} / m_{N^{*}}\right)^{2}$; in fact one can show from the equations of motion that only the convection current contribution should be. We also use effective masses for nucleons and deltas:

$$
\begin{aligned}
& m_{N}^{*}=m_{N}\left(1-C \rho / \rho_{0}\right) \\
& m_{\Delta}^{*}=m_{\Delta}-\left(m_{N}-m_{N}^{*}\right) .
\end{aligned}
$$

The factor $z_{\pi}$ in Eq. (9) accounts for effects of $\pi N$ s-wave interactions 18] which, in a scattering length approximation, is estimated to be

$$
z_{\pi}=(1+4 \pi \lambda \rho)^{-1 / 2}
$$

with $\lambda \simeq 0.11 \mathrm{fm}^{3}$.

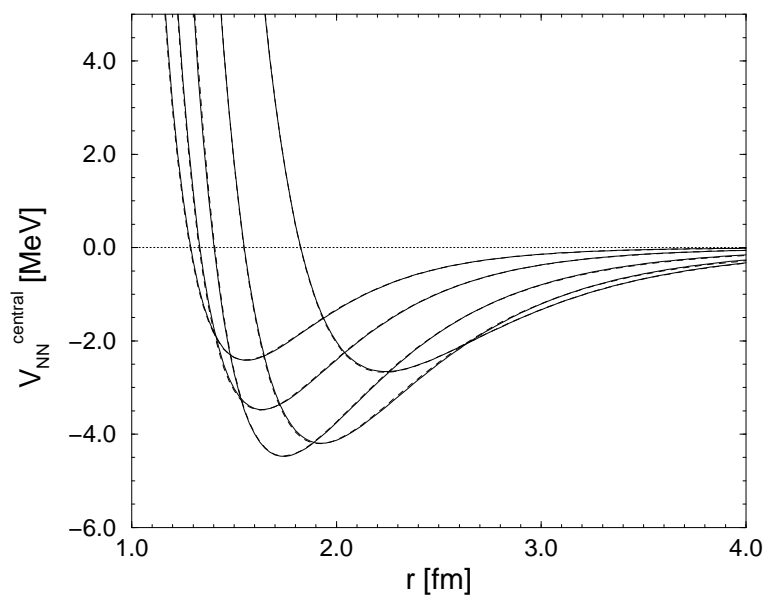

FIG. 1. In-medium central $N N$ potential, Eq. (5), supplemented by $\omega$ exchange (using $m_{\omega}^{*} / m_{\omega}=\Phi(\rho)$ ) for various nuclear matter densities. The solid lines are our microscopic results from in-medium correlated $2 \pi$ exchange and the dashed lines correspond to a fit with two zero-width sigma mesons with the parameters given in Table I. The densities are 0 , $0.5 \rho_{0}, \rho_{0}, 1.5 \rho_{0}$, and $2 \rho_{0}$ from top to bottom at the right side of the graph.

The resulting in-medium central $N N$ potential, Eq. (5), supplemented with zero-width $\omega(782)$ exchange (using $m_{\omega}^{*} / m_{\omega}=\Phi(\rho)$ ), is shown in Fig. 1. At low densities the increase in attraction in $V_{N N}^{2 \pi, \text { corr }}(r)$ dominates since both the softening of the pion dispersion relation and the enhanced attraction due to $t$-channel $\rho$ exchange with reduced mass result in a marked shift of spectral strength of the ' $\sigma$ ' (i.e. in $\eta_{00}\left(t^{\prime}\right)$ ) to lower energies. However, at higher densities this tendency becomes more and more suppressed: on the one hand, both the increasing $g^{\prime}$ and $z_{\pi}$ decelerate the pion softening, and on the other hand the contact interactions in the $\pi \pi$ kernel $V_{\pi \pi}$, being proportional to $\left(f_{\pi}^{*}\right)^{-2}$, balance the increase in attraction in $\rho$ exchange. As a result, the attraction from $V_{N N}^{2 \pi, \operatorname{corr}}(r)$ increases less rapidly than the repulsion in $V_{N N}^{\omega}(r)$ (due to a reduced $\omega(782)$ mass). That means that at low densities our microscopically calculated $2 \pi$ exchange potential behaves approximately like a scalar meson, with mass decreasing at the same rate as for the $\omega$. Approaching $\rho_{0}$, the contact terms dictated by chiral symmetry slow the decrease of the effective $\sigma$ mass, thus enabling saturation.

To investigate the quantitative impact on nuclear matter properties, we apply the in-medium $2 \pi$-exchange 
TABLE I. Density-dependent parametrization of the correlated in-medium $2 \pi$ exchange in terms of two zero-width sigma bosons $\left(\Lambda_{\sigma}=3 \mathrm{GeV}\right.$ in all cases $)$.

\begin{tabular}{ccccc}
\hline \hline density $\left(\rho_{0}\right)$ & $m_{\sigma_{1}}(\mathrm{MeV})$ & $g_{\sigma_{1}}^{2} / 4 \pi$ & $m_{\sigma_{2}}(\mathrm{MeV})$ & $g_{\sigma_{2}}^{2} / 4 \pi$ \\
\hline 0 & 640 & 4.36 & 377 & 0.640 \\
0.5 & 538 & 3.405 & 295 & 0.450 \\
0.75 & 471 & 2.875 & 249 & 0.309 \\
1.0 & 427 & 2.53 & 220 & 0.264 \\
1.25 & 395 & 2.35 & 200 & 0.234 \\
1.5 & 365 & 2.17 & 190 & 0.212 \\
2.0 & 315 & 1.765 & 181 & 0.162 \\
\hline \hline
\end{tabular}

model in a relativistic DBHF calculation. For this purpose, we start from the Bonn-B potential [20] which is a one-boson-exchange potential (OBEP) that includes the $\pi, \eta, \rho(770), \omega(782)$, and $a_{0}(980)$ mesons as well as a zerowidth $\sigma(550)$ boson and describes free-space $N N$ scattering accurately up to pion-production threshold. In this OBEP, we replace the zero-width $\sigma$ by our microscopic model of $2 \pi$ exchange explained above. To make the calculations more tractable, we parametrize $V_{N N}^{2 \pi, c o r r}(r ; \rho)$ in terms of two sharp scalar mesons with density-dependent masses and coupling constants, cf. Table I and dashed lines in Fig. 1. As discussed in the literature [21,22, the correlated $2 \pi$ exchange accounts for more than half of the intermediate-range attractiont. The remainder is provided by uncorrelated $2 \pi$ exchange which we parametrize, in our present calculations, in terms of a 'rest' $\sigma$ boson with less than half the coupling strength of the full $\sigma$ of a typical OBEP. This combination, plus the five mesons mentioned above ( $c f$. Table II) reproduces free-space $N N$ scattering as well as the original Bonn-B potential.

This model for the $N N$ interaction is now applied to nuclear matter. We use the DBHF approach [1],3], scale the nucleon mass as well as the meson and cutoff masses of $\rho(770)$ and $\omega(782)$ according to Eq. (7), and use the density-dependent correlated $2 \pi$ exchange. Our result for the energy per nucleon as a function of density is shown in Fig. 2 by the solid curve. The predicted saturation energy is $-15.1 \mathrm{MeV} /$ nucleon at a density corresponding to $k_{F}=1.32 \mathrm{fm}^{-1}\left(\rho=2 k_{F}^{3} / 3 \pi^{2}\right)$, in good agreement with the empirical values. The incompressibility, $K_{V}^{-1}$, is $356 \mathrm{MeV}$, which is slightly above the currently favored

TABLE II. Meson parameters for the one-boson-exchanges involved in the $N N$ model applied in the present work.

\begin{tabular}{cccccc}
\hline \hline & $J^{P}$ & $I$ & $m_{\alpha}(\mathrm{MeV})$ & $g_{\alpha}^{2} / 4 \pi$ & $\Lambda_{\alpha}(\mathrm{GeV})$ \\
\hline$\pi$ & $0^{-}$ & 1 & 138.03 & 14.6 & 1.2 \\
$\eta$ & $0^{-}$ & 0 & 548.8 & 5 & 1.5 \\
$\omega$ & $1^{-}$ & 0 & 782.6 & 20 & 1.5 \\
$\rho$ & $1^{-}$ & 1 & 769 & $0.95^{*}$ & 1.3 \\
$a_{0}$ & $0^{+}$ & 1 & 983 & 2.9908 & 1.5 \\
rest- $\sigma$ & $0^{+}$ & 0 & 517 & 2.8386 & 3.0 \\
\hline${ }^{*} f_{\rho} / g_{\rho}=6.1$ &
\end{tabular}

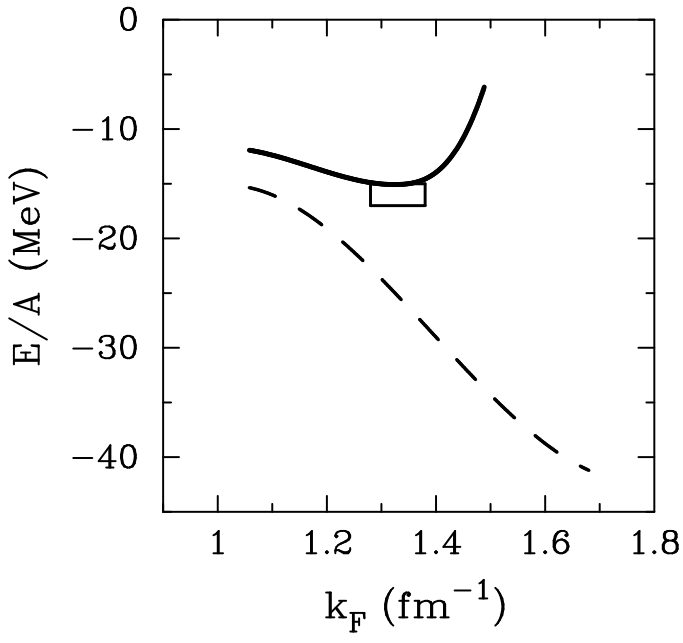

FIG. 2. Energy per nucleon in nuclear matter, $E / A$, as a function of density in terms of the Fermi momentum $k_{F}$. The solid curve displays our main result which is obtained from a DBHF calculation applying BR scaling, Eq. (7), to $\rho$ and $\omega$ and using the correlated in-medium $2 \pi$ exchange explained in the text. When the latter is replaced by a zero-width $\sigma$ boson which is subjected to BR scaling, then the dashed curve is obtained. The box represents the empirical values for nuclear matter saturation $\left(E / A=-16 \pm 1 \mathrm{MeV}, k_{F}=1.33 \pm 0.05\right.$ $\left.\mathrm{fm}^{-1}\right)$.

range. (Similar results have been obtained with a more schematic mean field model [23] in which introduction of more rapid scaling of the nucleon effective mass, $m_{N}^{*} / m_{n}=\sqrt{g_{A}^{*} / g_{A}} \Phi(\rho)$, with loop effects 酒, substantially decreased $K_{V}^{-1}$. We believe that it would do so in our case too, but because of greatly increased technical complications, we have not yet been able to go to higher loop order.)

Our results may be contrasted with a simpler calculation in which a conventional OBEP is applied and the $\sigma$ boson (besides $\rho$ and $\omega$ ) is subjected to BR scaling. As discussed, no saturation can be achieved in this case, as clearly revealed by the dashed curve in Fig. 2 .

In summary, we have studied the impact of medium modifications in meson exchanges of the $N N$ potential on saturation properties of nuclear matter. The twopion exchange contribution has been calculated microscopically with the standard many-body effects on the in-medium pion propagation taken into account. For the vector meson exchanges in both the $\pi \pi$ and the nucleonnucleon sector we assumed a universal decrease in their masses $m_{\rho, \omega}$, which has also been applied to the nucleon mass in the many-body parts of the calculation. We find that in a relativistic DBHF calculation this provides a reasonable saturation mechanism, thus improving earlier results in which a universal scaling of the exchanged meson masses (including the mass of an alleged ' $\sigma$ meson') could not achieve a stable configuration. The main reason for this difference is the fact that the combination 
of nuclear many-body effects on the pions and the shortrange repulsive part of a (broken) chirally symmetric $\pi \pi$ interaction suppresses the increase of attraction in the $\sigma$ channel at nuclear densities above the empirical saturation value. Thus the chiral constraints on the $\pi \pi$ interaction in our model [16] are seen to play a subtle, but crucial, role in nuclear matter saturation. Whether the mass scaling of the vector mesons, which plays an equally important role in the saturation process, can be understood in a similar (microscopic) way to that of the ' $\sigma$ ' remains a question for further investigation.

One of us (RR) acknowledges support from the Alexander-von-Humboldt foundation as a Feodor-Lynen fellow. This work was supported in part by the U.S. Department of Energy under Contract No. DE-FG0288ER40388 and by the U.S. National Science Foundation under Grant No. PHY-9211607.

[1] R. Machleidt, Adv. Nucl. Phys. 19, 189 (1989).

[2] F. Coester, S. Cohen, B. D. Day, and C. M. Vincent, Phys. Rev. C 1, 769 (1970).

[3] R. Brockmann and R. Machleidt, Phys. Rev. C 42, 1965 (1990).

[4] G. E. Brown and M. Rho, Phys. Rev. Lett. 66, 2720 (1991).

[5] A. Amorin and J.A. Tjon, Phys. Rev. Lett. 68, 772 (1992).

[6] G.E. Brown, H. Müther, and M. Prakash, Nucl. Phys. A506, 565 (1990).

[7] G. E. Brown and R. Machleidt, preprint SUNY-NTG-9230 and e-print archive nucl-th/9210010.

[8] Z. Aouissat, R. Rapp, G. Chanfray, P. Schuck and J. Wambach, Nucl. Phys. A581, 471 (1995).

[9] R. Rapp, J.W. Durso and J. Wambach, Nucl. Phys. A596, 436 (1996).

[10] H.-C. Kim, J. W. Durso and K. Holinde, Phys. Rev. C 49, 2355 (1994).

[11] C. Schütz, K. Holinde, J. Speth, B. C. Pearce and J. W. Durso, Phys. Rev. C 51, 1374 (1995),.

[12] D. Lohse, J. W. Durso, K. Holinde and J. Speth, Phys. Lett. B234, 235 (1989); Nucl. Phys. A516, 513 (1990); B.C. Pearce, K. Holinde and J. Speth, Nucl. Phys. A541, 663 (1992).

[13] S. Weinberg, Phys. Rev. Lett. 17, 616 (1966); Phys. Rev. 166 (1968) 1568.

[14] R. Rapp, PhD thesis Bonn 1996, in Berichte des Forschungszentrum Jülich 3195, (Jülich, 1996).

[15] G. Höhler, in Pion-Nucleon Scattering, ed. H. Schopper, Landolt-Börnstein, New Series Group I, Vol. 9b, Pt. 2 (Springer-Verlag, New York, 1983).

[16] R. Rapp, J.W. Durso and J. Wambach, Nucl. Phys. A615, 501 (1997).

[17] T. Hatsuda and S. H. Lee, Phys. Rev. C 46, R34 (1992).

[18] A.B. Migdal, E.E. Saperstein, M.A. Troitsky and D.N.
Voskresensky, Phys. Rep. 192, 179 (1990).

[19] G.E. Brown, M. Buballa, Zi Bang Li and J. Wambach, Nucl. Phys. A593, 295 (1995).

[20] The Bonn-B potential is presented in Ref. 11 (see, in particular, Appendix A, Table A.2, therein).

[21] J.W. Durso, A.D. Jackson, and B.J. Verwest, Nucl. Phys. A345, 471 (1980).

[22] R. Machleidt, K. Holinde, and C. Elster, Phys. Rep. 149, 1 (1987).

[23] C. Song, G.E. Brown, D.-P. Min and M. Rho, SNUTP 97-052 and e-print archive hep-ph/9705255. 